\title{
Incidence of SARS COV-2 \& Influenza A in SARI Patients Attending Tertiary Care Hospital, Thanjavur
}

\author{
D. Sivasankari, Jeyabharathi Jeyaraj Kathirvelan* and Eunice Swarna Jacob \\ Department of Microbiology, Orathanadu, Thanjavur - 614625, Tamil Nadu, India \\ *Corresponding author
}

\section{A B S T R A C T}

\section{Keywords}

Lower respiratory tracts, patients, pneumonia, World Health Organization

Article Info

Accepted: 25 May 2021 Available Online: 10 June 2021
The outbreak of corona virus disease 2019 (COVID-19) caused by the severe acute respiratory syndrome corona virus 2 (SARS-CoV-2) is currently the peak season of common respiratory viral infections. Influenza $\mathrm{A}$ was one of the most common respiratory viruses, which may have caused initial false negative results of real-time reverse-transcriptase polymerase chain reaction for severe acute respiratory syndrome corona virus 2 (SARS-CoV-2) among the SARI patients. In contrast, clinicians cannot rule out SARS-CoV-2 and Influenza infection based on the clinical and laboratory findings. Therefore, clinicians must have a high index of suspicion for coinfection among COVID-19 patients. After recognizing the possible pathogens causing coinfection among COVID-19 patients, appropriate antimicrobial agents can be recommended. A total of 104 SARI patients with clinically suspected of COVID-19 in a tertiary care hospital Thanjavur were recruited from December 2020 to February 2021. Nasopharyngeal swabs, throat swabs were collected to detect SARS-CoV-2 and Influenza A virus by using real-time reverse transcription-polymerase chain reaction (RT-PCR). Clinical characteristics and laboratory test findings were acquired from medical records. All data were analysed to recognize the epidemiological patterns. Among the 104 SARI patients, 25.6\%(27/104) patients with suspected COVID-19 were eventually confirmed to have SARS-CoV-2 infection, and the most frequently observed symptoms were cough $(86 \%, 90 / 104)$ followed by Fever $(84 \%, 88 / 104)$, breathlessness $(80 \%, 83 / 104)$, myalgia $(25 \%, 27 / 104)$, vomiting $(15.3 \%, 16 / 104)$, diarrhoea (12.5\%13/104), chest pain $(10.5 \%, 11 / 104)$. There were no cases out of 104 SARI patients who were positive for Influenza (H1N1) by RT-PCR.

\section{Introduction}

Since December 2019, there has been a cluster of patients with pneumonia of previously unknown cause in Wuhan, China. Research by the Chinese Centre for Disease Control and
Prevention assessed the lower respiratory tracts of these patients and discovered a novel corona virus, which has since been named the 2019 novel corona virus ${ }^{15}$. On February 11, 2020, the World Health Organization officially named this novel corona virus 
pneumonia as coronavirus disease 2019 (COVID-19), whereas the International Committee on Taxonomy of Viruses has named it severe acute respiratory syndrome corona virus 2 (SARS-CoV-2) ${ }^{15}$. Approximately a decade ago, the World Health Organization (WHO) declared the first of two global epidemics: on June 11, 2009, an influenza A H1N1 pandemic (pH1N1); the second and current pandemic of corona virus disease 2019 (COVID-19) was declared on March 11, 2020.

The two pandemics of the 21st century originated from different viruses, but they have some similarities, such as they were both caused by enveloped RNA viruses, usually of spherical morphology ${ }^{1}$. Other points that call attention are related to the frequent mutations and diversity of the hosts that can be infected ${ }^{1}$.Infections of the respiratory system, such as influenza-like illness, cold, bronchitis and pneumonia are the main causes of morbidity and mortality worldwide. SARSCOV-2 \&Influenza viruses are the most common pathogens of respiratory tract infections causing high morbidity and mortality ${ }^{1}$.

Corona virus disease 2019 (CoVID-19) is a new infectious disease caused by severe acute respiratory syndrome coronavirus 2 (SARSCoV-2), which originated from Wuhan in China and has now spread globally. However, despite the concern focused on SARS-CoV-2, influenza virus continues to circulate and cause disease. Physicians should be alert that a positive test for COVID-19 does not rule out the possibility of Influenza virus disease.

For H1N1, the incubation period was 1-3 days, but sometimes it extended to 7 days. For COVID-19, the incubation period is usually longer (2-14 days), with an average of 5.2 days ${ }^{1}$. Among the H1N1 and COVID-19 cases, there are similarities in the clinical spectra. The clinical picture usually tends to start with fever and cough, sometimes accompanied by a sore throat and myalgia ${ }^{1}$. For the laboratory diagnosis of Influenza A and SARS-CoV-2, clinical samples including throat or nasopharyngeal swab, a saliva sample or aspirate of the lower respiratory system which are collected during the acute phase of the disease ${ }^{1}$. Another option for detecting SARS-CoV-2 includes diagnostic imaging, which is obtained by computed tomography of the chest ${ }^{1}$.The typical findings from chest CT scans were bilateral groundglass opacity and sub segmental areas of consolidation ${ }^{2}$.

During the SARS pandemic in 2003, Yang et al., found that the patients with fever, cough or sore throat had a $5 \%$ of Influenza virus positive rate $^{3}$. This raises the concern that there might be co-infection or super-infection of seasonal influenza and the novel coronavirus. Measures should be taken to enhance the respiratory infectious diseases surveillance systems and screen the people with fever, cough or sore throat for both viruses with oropharyngeal and nasopharyngeal swabs. Hence we planned to conduct a cross sectional study to find the incidence of SARS-COV-2 and H1N1 in SARI patients admitted in the Tertiary Care Hospital at Thanjavur.

\section{Materials and Methods}

This was a cross sectional study investigated on the epidemic situation in the SARI patients who attended Tertiary care hospital at Thanjavur from December 2020 to February 2021

\section{Eligibility criteria}

Patients included in this study were those with fever $\left(\geq 37.0^{\circ} \mathrm{C}\right)$, respiratory symptoms (e.g., cough, sputum production, haemoptysis, shortness of breath, wheezing, and chest pain, etc.) and other symptoms like myalgia, 
vomiting, diarrhoea etc.

\section{Data collection}

Demographic and clinical data of the patients were entered into the case report form. The data included the following: demographic characteristics (age and sex), underlying diseases, co morbidities, clinical symptoms (fever, cough, sputum, dyspnoea, chest pain, rashes, nausea, vomiting, abdominal pain, diarrhoea, and headache), signs (body temperature, heart rate, respiratory rate, and BP), laboratory tests (blood routine test, arterial blood gas analysis, and blood chemistry), /images of the lung (chest CT scan). Anti-microbial therapy, respiratory support and complications were also recorded.

\section{Ethical committee clearance}

This study was approved by the National Ethical Committee Registry for Biomedical and health research (No:EC/NEW/INST/ 2020/1058). Informed consent was obtained from all the participants or their parents/caregivers before sample collection.

\section{Sample collection and processing}

Nasopharyngeal swabs and throat swabs were collected from patients with clinically suspected severe acute respiratory infections ${ }^{2}$. After sampling, the specimens were placed into collection tubes filled with a $2 \mathrm{ml}$ viral transport medium and transported immediately to the central laboratory at a temperature of 4$8^{\circ} \mathrm{c}$. Details about the patients like the name $\&$ the contact details were not known to the laboratory personnel who performed the processing and nucleic acid extraction. Total viral RNA was extracted using Helini viral RNA extraction kit (Helini biomolecules, Chennai). Briefly, $200 \mu$ of each specimen was transferred into a tube containing $210 \mu 1$ lysis solution and $15 \mu \mathrm{l}$ magnetic beads, followed by incubation at $56{ }^{\circ} \mathrm{C}$ for $10 \mathrm{~min}$ with vigorous shaking. The obtained cell lysates were transferred to a collection tube and then kept inside the automated RNA extractor. The final step was the bound RNA extracts were eluted to a final volume of $100 \mu 1$ with the elution buffer.

\section{SARS-CoV-2 real-time quantitative PCR}

Real-time reverse transcription-polymerase chain reaction (RT-PCR) was performed by amplifying two target genes, including RNA dependant RNA polymerase (RDRP)and Nucleocapsid protein (N). The test was performed according to Labgun RT-PCR kitprotocol (labgun ${ }^{\mathrm{TM}}$ covid -19 Exofast RTPCR kit by Siemens healthcare Pvt Ltd). Each reaction mixture contained $4 \mu 1$ of one-step RT-PCR reaction buffer, $2 \mu$ l of enzyme mix, $4 \mu 1$ of assay, $5 \mu \mathrm{l}$ of RNAse free water and $5 \mu 1$ of RNA as a template. Thermal cycling was initiated at $50^{\circ} \mathrm{C}$ for $20 \mathrm{~min}$ (for reverse transcription), followed by $95^{\circ} \mathrm{C}$ for $10 \mathrm{~min}$ (for annealing) and 35cycles of amplification at $95^{\circ} \mathrm{C}$ for $15 \mathrm{~s}$ and $60^{\circ} \mathrm{C}$ for $30 \mathrm{~s}$ in the CFX96 Touch RT-PCR system (BIO-RAD Laboratories).

\section{Influenza A real time quantitative PCR}

Real-time reverse transcription-polymerase chain reaction (RT-PCR) was performed by amplifying two target genes, Universal Inf A and H1N1 gene. Real-time RT-PCR assays were performed according to the Helini RTPCR kit protocol (HELINI SWINE FLU[H1N1] real time PCR Kit by HELINI Biomolecules, Chennai, INDIA. Each reaction mixture contained $8 \mu$ lone step master mix, 2 $\mu 1$ Taq enzyme mix, $5 \mu 1$ H1N1 Primer Probe mix and $10 \mu$ lof RNA as a template. Thermal cycling was initiated at $50^{\circ} \mathrm{C}$ for $20 \mathrm{~min}$ (for reverse transcription), followed by $95^{\circ} \mathrm{C}$ for $15 \mathrm{~min}$ and 45 cycles of $95^{\circ} \mathrm{C}$ for $20 \mathrm{~s}, 56^{\circ} \mathrm{C}$ for $20 \mathrm{~s}$ and $72^{\circ} \mathrm{C}$ for $20 \mathrm{~s}$ in the Light cycler96 (Roche). 


\section{Results and Discussion}

In total, 104 individuals admitted with suspicious symptoms of SARI were included. Of these the average age was 52.9 years, most patients were in the age group 51-60(34.6\%) and $73 \%(76 / 104)$ were men. Cough (86\%, 90/104)was the most frequent symptom followed by Fever (84\%, 88/104), breathlessness $\quad(80 \%, 83 / 104), \quad$ myalgia $(25 \%, 27 / 104), \quad$ vomiting $(15.3 \%, 16 / 104)$, diarrhea (12.5\%13/104), chest pain $(10.5 \%, 11 / 104)$. Twenty-seven patients were confirmed to have COVID-19, with a positive rate of 25.9\%. Among the 104 SARI patients none of them were positive for Influenza (H1N1) by RT-PCR. Abnormal radiographic findings(ground glass opacities pertinent to covid findings) ${ }^{15}$ were observed on the chest computed tomography scans in most patients $(25.9 \%, 27 / 104)$ with positive rate of $96 \%$.

COVID-19 was declared pandemic by WHO on March 11 2020. Meanwhile, it was also the peak season for respiratory tract infections caused by Influenza virus, and their severe clinical symptoms and cross-species transmission patterns pose a huge threat to human health. However, it is difficult to distinguish clinically between the patients with SARIs and COVID-19, since the clinical manifestations are nearly the same. This raised the suspicion of possible H1N1 infection among the SARI patients. The influenza viruses and SARS-CoV-2 are both efficient in causing respiratory disease because they easily spread among humans through oral and nasal droplets $^{1}$. In patients with COVID-19, blood tests typically show leukopenia and lymphopenia and most chest computed tomography scans show ground-glass opacity and consolidation with bilateral lung involvement. Ground-glass opacities were more common in patients with COVID-19 than in patients with H1N1 $(\mathrm{P}<.001)^{15}$. Due to the knowledge generated from combating the circulation of different strains of seasonal influenza, antiviral treatment was already well established, such as the use of Oseltamivir and Zanamavir ${ }^{1}$.In contrast to $\mathrm{pH} 1 \mathrm{~N} 1$, the COVID-19 pandemic did not have an antiviral drug or vaccine available initially ${ }^{1}$.

During the SARS pandemic in 2003, Yang et al., found that the patients with fever, cough or sore throat had a $5 \%$ of influenza virus positive rate, and with SARS infection reportedly increasing at the meantime ${ }^{1}$. Although the aetiology of SARI are common respiratory viruses (Influenza, RSV, Metapneumovirus etc) they may be the leading cause of the disease. Hence we should see for other viral pathogens. This may help to correctly handle other respiratory tract infections. Hence we conducted a cross sectional study in a Tertiary care hospital at Thanjavur during the period of December 2020 to February 2021.

In this study average age of the SARI patients was 52.9 with $34.6 \%$ patients in the age group of 51-60 years and $73.1 \%$ were male patients.

The co-morbid illness associated with SARI were Diabetes (28/104,26.9\%), Hypertension (16/104,15.3\%), CAD (8/104,7.7\%), tuberculosis $(7 / 104,6.7 \%)$, CKD $(5 / 104,4.8 \%)$ and $\operatorname{CaCx}(1 / 104,0.96 \%)$. All the 104 patients included in this study were tested for COVID19, Influenza A and H1N1. Out of the 104 patients tested only 27 were RT-PCR positive for COVID-19 with a positive detection rate of $25.6 \%$. 
Table.1 Age wise distribution of SARI patients

\begin{tabular}{|c|c|c|}
\hline Age of the patient & $\begin{array}{c}\text { Number of SARI } \\
\text { Patients (n) }\end{array}$ & Percentage (\%) \\
\hline$<\mathbf{2 0}$ & 3 & 2.8 \\
\hline $\mathbf{2 1 - 3 0}$ & 5 & 4.8 \\
\hline $\mathbf{3 1 - 4 0}$ & 16 & 15.3 \\
\hline $\mathbf{4 1 - 5 0}$ & 20 & 19.2 \\
\hline $\mathbf{5 1 - 6 0}$ & 36 & 34.6 \\
\hline $\mathbf{6 1 - 7 0}$ & 16 & 15.3 \\
\hline $\mathbf{7 1 - 8 0}$ & 4 & 3.8 \\
\hline$>\mathbf{8 0}$ & 4 & 3.8 \\
\hline Total & 104 & 100 \\
\hline
\end{tabular}

Table.2 Sex wise distribution of SARI patients

\begin{tabular}{|c|c|c|}
\hline Sex of the patients & No of SARI patients & Percentage \\
\hline Male & 76 & 73.1 \\
\hline Female & 28 & 26.9 \\
\hline Total & 104 & 100 \\
\hline
\end{tabular}

Table.3 Positivity rate of COVID-19 and H1N1 among the SARI patients

\begin{tabular}{|c|c|c|}
\hline & COVID 19 & H1N1 \\
\hline Number of SARI patients & 104 & 104 \\
\hline Positive cases & 27 & 0 \\
\hline Percentage & $25.9 \%$ & $0 \%$ \\
\hline
\end{tabular}

Table.4 Comparison of RT-PCR with CT scan

\begin{tabular}{|c|c|c|}
\hline & COVID 19 & H1N1 \\
\hline RT-PCR POSITIVE CASES & 27 & 0 \\
\hline $\begin{array}{c}\text { CT SCAN POSITIVE } \\
\text { CASES }\end{array}$ & 26 & 0 \\
\hline POSITIVITY & $96 \%$ & 0 \\
\hline
\end{tabular}


Fig.1 Age wise distribution of SARI patients

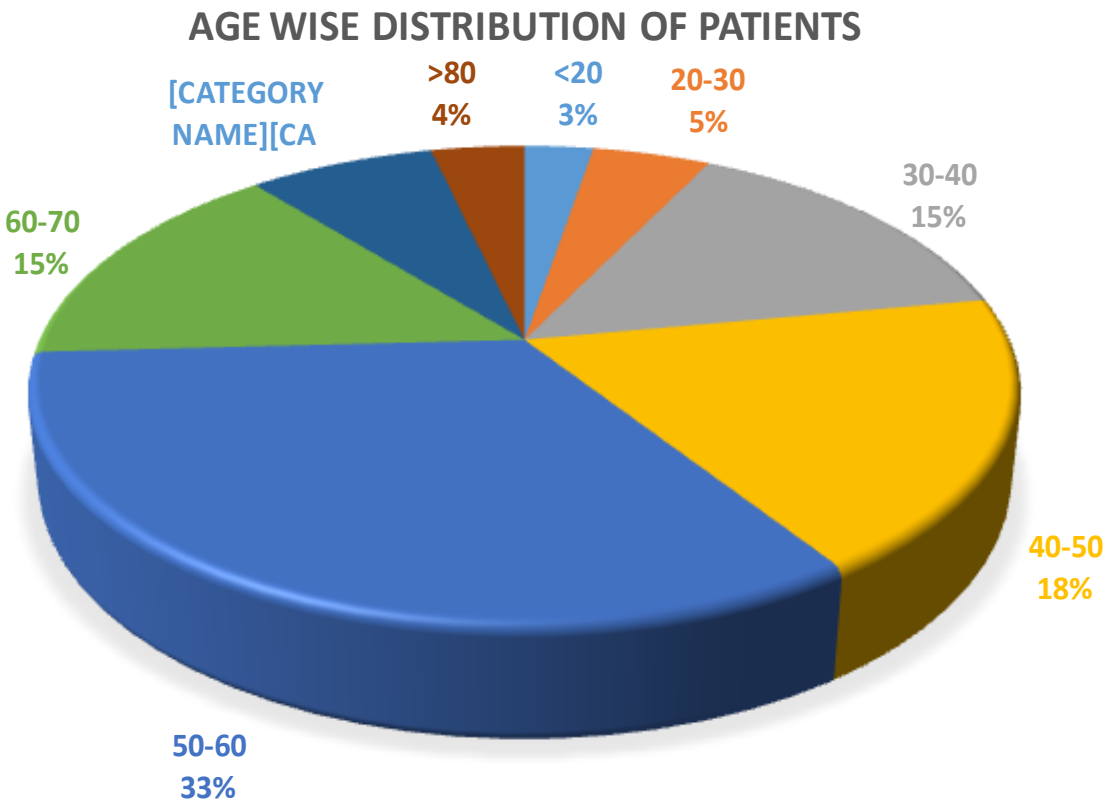

Fig.2 Sex wise distribution of SARI patients

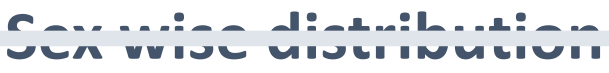

Number of patients Percentage

76

$73 \%$

28

[VALUE][

Male

Female 
Fig.3 Positivity rate of COVID-19 and H1N1 among the SARI patients

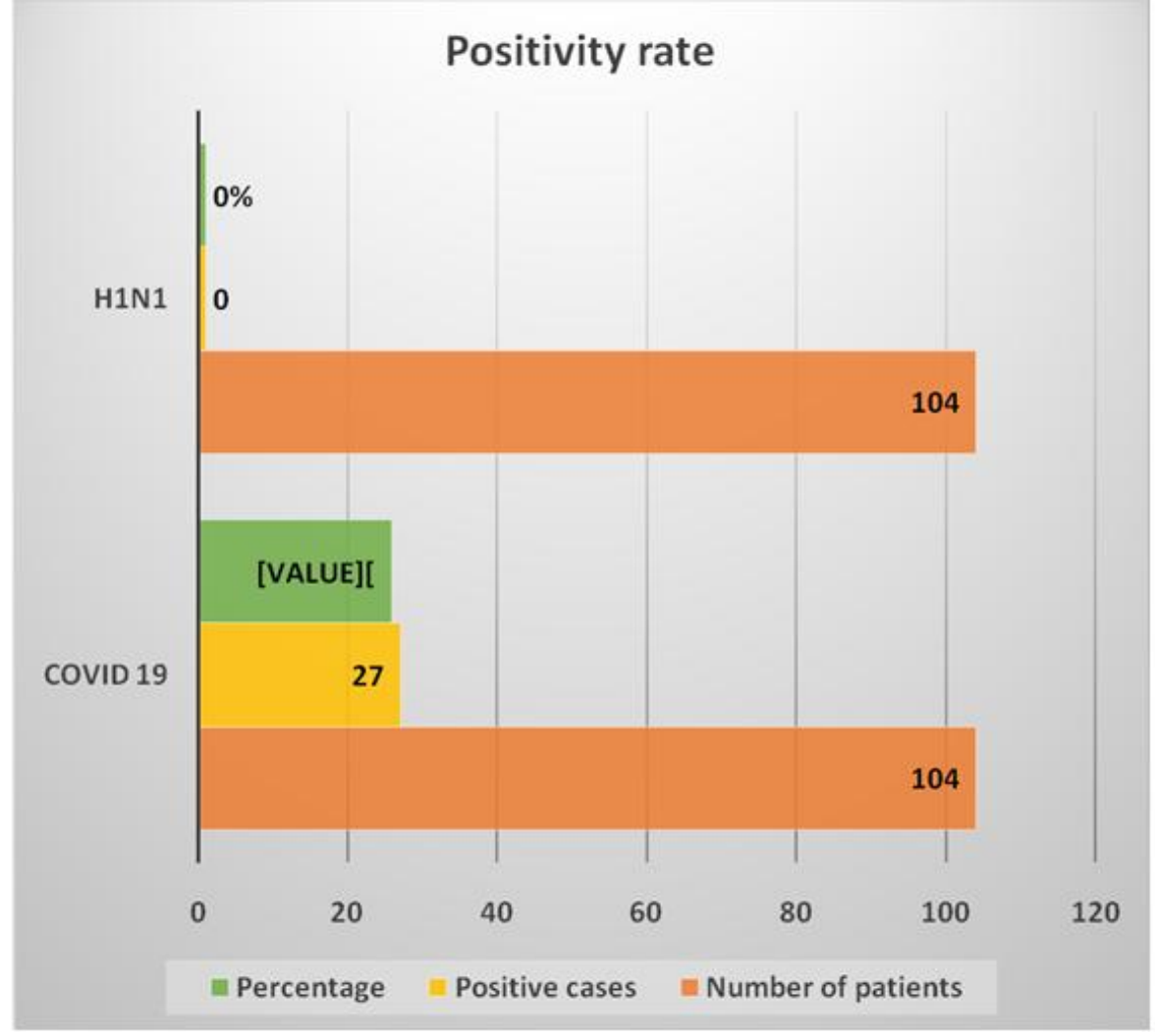

Fig.4 Comparison of RT-PCR with CT scan

\section{COMPARISION OF RT-PCR WITH CT SCAN}

no of SARI patients a RT-PCR positive $\quad$ CT scan positive

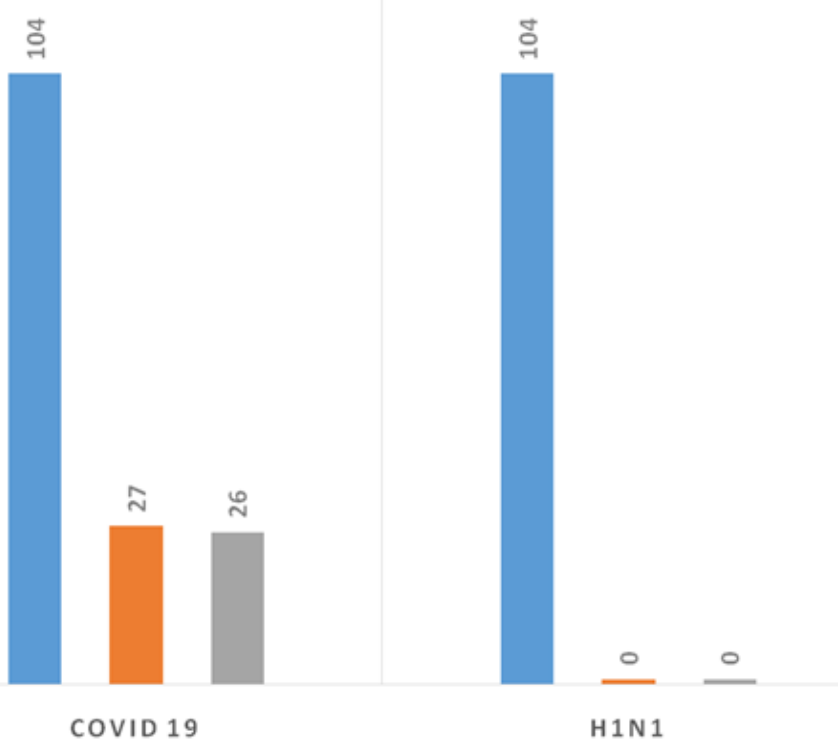


In the study by $\mathrm{Si}$ et al., $10.3 \%$ of the suspected COVID-19 was positive for other respiratory viruses ${ }^{2}$.In the study conducted by Kong et al., ${ }^{16}$ showed that the positive rate of rhinovirus/enterovirus was $4.8 \%$ among 806 adult patients with ARIs in Shanghai. Eight studies reported viral co-infections; rhinovirus/enterovirus and influenza $\mathrm{A}$ were the commonest co-pathogen, and corona virus, respiratory syncytial virus, parainfluenza, metapneumovirus, and influenza B virus were also reported as co-pathogens. But in our study there were no Influenza A positive cases confirmed by RT-PCR among the SARI patients.

In our study 26 patients out of 27 have radiographic findings pertinent to Covid-19 with a positive predictive value $96 \%$. Hence CT scan can be used along with RT-PCR to improve the sensitivity of the test for correct identification and treatment of SARI patients. Severity of the illness can also be assessed by the CT scan and blood investigations. Among the 104 SARI patients only 27 cases were positive for COVID -19, so there is possibility of infection with other respiratory pathogens. In our study we have tested the samples for universal Influenza $A$ and $\mathrm{H} 1 \mathrm{~N} 1$, although there was no positivity for Influenza A there may be other respiratory pathogens (Metapneumovirus, RSV etc) as the causative agent. Clinicians cannot rule out co-infection with other respiratory pathogens when diagnosing SARS-CoV-2 infection clinically. However, our findings were based on a limited number of samples. Further large-sample, well-designed studies including the other respiratory pathogens are warranted to investigate the prevalence of COVID-19 coinfection, risk of co-infection, microbiological distribution, and impact of co-infection on the clinical outcomes of COVID-19 patients. After obtaining the data regarding the infections with other viral respiratory pathogens, empirical antimicrobial agents in suspected COVID-19 cases among the SARI patients can be recommended.

\section{References}

1. Dacosta, V. G. et al., Comparative epidemiology between the 2009 H1N1 influenza and COVID-19 pandemics, Journal of Infection and Public Health 13 (2020) 1797-1804

2. Epidemiological surveillance of common respiratory viruses in patients with suspected COVID-19 in Southwest China, Si et al., BMC Infectious Diseases (2020) 20:688

3. Co-infections among patients with COVID19: The need for combination therapy with non-anti-SARS-CoV-2 agents, chih-cheng lai et al., Journal of Microbiology(2020) 53, 505-512

4. Ding Q, Lu P, Fan Y, Xia Y, Liu M. The clinical characteristics of pneumonia patients co-infected with 2019 novel coronavirus and influenza virus in Wuhan, China. J Med Virol 2020 Mar 20. https://doi.org/10.1002/jmv.25781.

5. Wu X, Cai Y, Huang X, Yu X, Zhao L, Wang $\mathrm{F}$, et al., Co-infection with SARS-CoV-2 and influenza A virus in patient with pneumonia, China. Emerg Infect Dis 2020 Mar 11.

6. Xing Q, Li G, Xing Y, Chen T, Li W, $\mathrm{Ni} \mathrm{W}$, et al., Precautions are needed for COVID-19 patients with coinfection of common respiratory pathogens. MedRxiv 2020 Mar 5.

7. Co-infection with COVID-19 and influenza $\mathrm{A}$ virus in two died patients with acute respiratory syndrome, Bojnurd, Iran, J Med Virol. 2020;1-3 DOI: $10.1002 / j m v .26014$

8. Khodamoradi Z, Moghadami M, Lotfi M. Co-infection of coronavirus disease 2019 and Influenza: a report from Iran. Arch Iran Med. 2020; 23(4):239-243. 
Emerging Infectious Diseases • www.cdc.gov/eid - Vol. 26, No. 6, June 2020

9. World Health Organization. Novel coronavirus-China. Disease outbreak news: update 12 January [cited 2020 $\mathrm{Feb}$ 12]. https://www.who.int/csr/don/12january-2020-novel coronaviruschina/en

10. Wang D, Hu B, Hu C, Zhu F, Liu X, Zhang J, et al., Clinical characteristics of 138 hospitalized patients with 2019 novel coronavirus-infected pneumonia in Wuhan, China. JAMA. 2020 Feb 7 [Epub ahead of print]. https://doi.org/10.1001/ jama.2020.1585

11. Coinfection of Influenza Virus and Severe Acute Respiratory Syndrome Coronavirus 2 (SARS- COV-2), Di $\mathrm{Wu}$ et al., The Paediatric Infectious Disease Journal • Volume 39, Number 6, June 2020

12. Co-infections of SARS-CoV-2 with multiple common respiratory pathogens in infected patients, Dachuan Lin, D., et al., Sci China Life Sci

13. Coinfection with SARS-CoV-2 and influenza A virus Kondo $\mathrm{Y}$, et al., BMJ Case Rep 2020;13: e236812. doi:10.1136/bcr-2020-236812

14. Richardson S, Hirsch J S, Narasimhan $\mathrm{M}$, et al., Presenting characteristics, comorbidities, and outcomes among 5700 patients hospitalized with COVID-19 in the New York City area. JAMA 2020 doi:10.1001/jama.2020.6775.

15. Comparison of Hospitalized Patients with ARDS Caused by COVID-19 and H1N1 Xiao Tang et al., CHEST 2020; 158(1):195-205,chest journal.org.

16. Kong D C, Wu H Y, Zheng Y X, Pan $\mathrm{H}$, Jiang C Y, Zhang X, Chen J, Wu F. Etiologic and epidemiologic features of acute respiratory infections in adults from Shanghai, during 2015-2017. Zhonghua liu xing bing xue za zhi $=$ Zhonghua liuxingbingxue zazhi. 2019;40(8):904-10.

\section{How to cite this article:}

Sivasankari, D., Jeyabharathi Jeyaraj kathirvelan and Eunice Swarna Jacob. 2021. Incidence of SARS COV-2 \& Influenza A in SARI Patients Attending Tertiary Care Hospital, Thanjavur. Int.J.Curr.Microbiol.App.Sci. 10(06): 688-696. doi: https://doi.org/10.20546/ijcmas.2021.1006.076 